\title{
Hat der Arbeitsmarkt wirklich von Hartz IV profitiert?
}

Ernst Niemeier

Das Institut für Arbeitsmarkt- und Berufsforschung (IAB) hat eine positive Bilanz $z^{1}$ der Wirkung des Sozialgesetzbuches II (Hartz IV) gezogen. Dies ist aus verschiedenen Gründen überraschend: Die Wirkungen der positiven konjunkturellen Entwicklung werden de facto völlig vernachlässigt. Die Rede ist nur von positiven Wirkungen von Hartz IV beim angeblichen Abbau struktureller Arbeitslosigkeit. Dass der Abbau der Arbeitslosigkeit in den Jahren 2006 bis 2008 aber wahrscheinlich primär der Nachfragedynamik zu verdanken ist, die durch die langjährige weltwirtschaftliche Entwicklung und durch die in der Folge steigende Investitionsnachfrage bestimmt wurde, lässt sich schon erkennen, wenn man das Okun'sche Gesetz beachtet, das den Zusammenhang zwischen dem Produktionswachstum (Bruttoinlandsprodukt, BIP) und der Veränderung der Arbeitslosenquote wiedergibt: Vereinfacht gesprochen ist die Veränderung der Arbeitslosenquote eine Funktion der Differenz zwischen der Wachstumsrate der Produktion (des BIP) und der sogenannten normalen Wachstumsrate. Die „normale Wachstumsrate der Produktion“ ist erforderlich, um den Stand der Arbeitslosigkeit unverändert $\mathrm{zu}$ lassen. Sie wird entscheidend durch die Produktivitätssteigerung bestimmt. Wenn die tatsächliche Wachstumsrate höher liegt als die Produktivitätssteigerung, sinkt die Arbeitslosigkeit. Vergleicht man das Produktionswachstum und die Produktivitätssteigerungen der beiden letzten Aufschwungphasen, also der Jahre 1998 bis 2000 und der Jahre 2006 bis 2008, wird deutlich, dass die Arbeitslosigkeit in der Zeit von 2006 bis 2008 stärker sinken musste als in der vorhergehenden Aufschwungphase, weil das BIP abzüglich der „normalen Wachstumsrate“ (Produktivitätssteigerung) stärker gestiegen ist als
1998 bis 2000, nämlich um 3,52 \% statt um 2,12\%. ${ }^{2}$ Dabei spielte allerdings auch die gesunkene Produktivitätssteigerung eine Rolle. Dass die Arbeitslosigkeit in der letzten Phase stärker sank, hat zusätzliche Ursachen im demografisch bedingten Rückgang des Arbeitsangebots.

Peter Bofinger hat in seinem Minderheitenvotum zum Jahresgutachten 2007/08 des Sachverständigenrats überzeugend aufgezeigt, dass die Arbeitsmarktreformen (Hartz-Gesetze) die Arbeitslosigkeit nicht spürbar vermindert haben. ${ }^{3}$ Diese Bewertung wird durch das Okun'sche Gesetz bestätigt.

Die bisherigen Argumente schließen nicht aus, dass die Hartz-Reformen einen kleinen positiven Effekt gehabt haben. Die Analyse dieser Arbeitsmarktreformen schließt jedoch aus, dass sie die Arbeitslosigkeit spürbar positiv beeinflusst haben können:

(1) Die Ursachendiagnose für die bestehende Arbeitslosigkeit, die Hartz IV zugrunde liegt, ist falsch, weil das unterstellte menschliche Verhalten unrealistisch ist.

(2) Das Konzept von Hartz IV unterstellt fälschlich, dass ausreichend viele offene Stellen verfügbar waren. Sie sind aber eine notwendige Bedingung für den Erfolg.

(3) Strukturelle Arbeitslosigkeit ist nur schwer von konjunktureller Arbeitslosigkeit zu unterscheiden, sodass selbst vermeintlich strukturelle Arbeitslosigkeit unter Umständen durch nachfragepolitische Maßnahmen bekämpft werden kann.

(4) Die Beveridge-Kurve, mit der in der IAB-Untersuchung eine Verminderung der strukturellen Arbeitslosigkeit begründet wird, zeigt kaum eine Abnahme dieser Arbeitslosigkeit an.

\section{FALSCHE URSACHENDIAGNOSE DER ARBEITSLOSIGKEIT}

All jene Ökonomen, die eine positive Wirkung von Hartz IV auf den Arbeitsmarkt zu erkennen glauben (und direkt oder indirekt an der Konzeption von Hartz mitgewirkt haben), gehen davon aus, dass die deutsche Arbeitsmarktentwicklung im Vergleich zur Entwicklung in anderen westlichen Ländern auf ein besonderes deutsches strukturelles oder institutionelles Problem hinweise. Während sich in den westlichen Ländern die Arbeitslosigkeit in einer Aufschwungphase wieder auf das ursprüngliche Niveau zurückgebildet habe, sei der Sockel der Arbeitslosigkeit in Deutschland stetig gestiegen. Dieser augenfällige Unterschied kann nach Meinung der genannten deutschen Ökonomen nur durch die Arbeitsmarktinstitutionen bedingt sein. Die Institutionen bewirkten eine zögerliche Bereitschaft der Arbeitslosen, Arbeitsplätze anzunehmen. Deshalb seien Agenda 2010 und Hartz-Reformen notwendig gewesen. Zwar gibt es unbestritten

1 Möller, J./Walwei, U./Koch, S./Kupka, P./Steinke, J. (2009): Fünf Jahre SGB II: Eine IAB-Bilanz. Der Arbeitsmarkt hat profitiert, IAB-Kurzbericht 29, Nürnberg

2 Ermittelt aus Tabellen 12 (S. 362) und 14 (S. 364), in: Sachverständigenrat zur Begutachtung der gesamtwirtschaftlichen Entwicklung (SVR) (2009): Die Zukunft nicht aufs Spiel setzen.

3 Sachverständigenrat zur Begutachtung der gesamtwirtschaftlichen Entwicklung (SVR) (2007): Das Erreichte nicht verspielen, S. 343

Ernst Niemeier, Dr., war bis zum Wintersemester 2008/2009 Lehrbeauftragter für Wirtschaftswissenschaften an der Hochschule Bremen und lebt jetzt als freier Publizist in der Nähe von Hamburg. e-mail: ernst.niemeier@t-online.de 
auch die zögerlichen Verhaltensweisen, die die Hartz-IV-Ökonomen unterstellen. Sie können das Massenphänomen Arbeitslosigkeit aber nicht erklären.

Der Vergleich der deutschen Entwicklung mit der westlicher Länder lässt die unterschiedlichen makroökonomischen Politiken als entscheidende Ursache allerdings außer Acht. Der amerikanische Wirtschaftsnobelpreisträger Robert Solow hat diese Ursachen für die unbefriedigende deutsche Entwicklung in einem Interview im Jahre 2007 besonders prägnant benannt: „Die Diskussion ist auf Arbeitsmarktreformen beschränkt. (...) Es gibt keine Diskussion über eine echte, makroökonomische Wirtschaftspolitik, anders als in den USA und in Großbritannien. (...) Außerdem war die Geld- und Finanzpolitik schon immer prozyklisch. Wenn die Wirtschaft wächst, wird stimuliert. Und wenn die Wirtschaft stagniert, wird gespart, statt mit einer expansiveren Finanzpolitik zu helfen. Und die Geldpolitik ist übertrieben streng. Schon die Bundesbank hat nie Verantwortung für Produktion und Beschäftigung übernommen, sondern war immer nur darauf aus, die Inflation zu drücken. Die Europäische Zentralbank macht so weiter". ${ }_{4}^{4}$ Diese schon seit den 1980er Jahren falsch betriebene makroökonomische Politik $^{5}$ in Deutschland kann den Anstieg des Sockels der Arbeitslosigkeit erklären, ohne auf ominöse institutionelle Ursachen zurückgreifen zu müssen. Es zeigt sich also, dass die Problemanalyse falsch war, die den Hartz-Reformen zugrunde lag.

\section{ANALYSE DES HARTZ IV-KONZEPTES}

Abgesehen von den an sich begrüßenswerten Förderungsabsichten der Hartz-Gesetze, die nach verbreiteter Meinung unzulänglich praktiziert wurden und die vor allem nicht die notwendigen Arbeitsplätze schaffen können, bestand das Hauptziel dieser aktivierenden Arbeitsmarktpolitik darin, die „erwerbsfähigen Hilfsbedürftigen zu eigenverantwortlichem und autonomem Handeln zu befähigen". 6

Die Mittel der aktivierenden Arbeitsmarktpolitik, die die Hartz-Gesetze vor allem einsetzen, sind die Kürzung der Bezugsdauer des Arbeitslosengeldes, die Streichung der lohnabhängigen Arbeitslosenhilfe und deren Ersetzung durch eine verschärft bedürftigkeitsabhängige Sozialhilfe (Arbeitslosengeld II) sowie die Verschärfung der Zumutbarkeitsbedingungen für die
Annahme eines Arbeitsplatzes hinsichtlich Tätigkeitsart, Entgelt und Entfernung zur Arbeitsstätte. Diese Maßnahmen - euphemistisch Reformen genannt - entfalten einen erheblichen Druck auf die Arbeitslosen, angebotene Arbeitsplätze anzunehmen. Voraussetzung dafür, dass eine positive Arbeitsmarktwirkung hätte eintreten können, wäre aber erstens gewesen, dass Arbeitslose nur durch Druck zur Arbeitsplatzannahme hätten „gezwungen“ werden können. Behauptet man, dass mit der Verminderung der Zahl der ,erwerbsfähigen Hilfsbedürftigen" um 519.185 vom Juni 2006 bis zum Juni $2009^{7}$ „die Ausrichtung der Grundsicherungsleistungen an der Aufnahme einer Erwerbsarbeit... zumindest in der Summe durchaus messbare Erfolge gezeitigt ${ }^{\text {"8 }}$ habe, dann unterstellt man, dass mindestens eine halbe Million ALG-II-Bezieher nicht eigenverantwortlich und autonom handelten; dass sie nur unter Druck der Hartz-IVRegelungen zur Arbeitsaufnahme hätten bewegt werden können. Arbeitslosigkeit kommt nach dieser fragwürdigen Behauptung also vor allem durch Arbeitsunwilligkeit der Arbeitslosen zustande.

Ist eine solche Annahme in einer Gesellschaft realistisch, zu deren zentralen Werten die Arbeit zählt? Nicht nur der gesunde Menschenverstand hält diese Annahme für unglaubwürdig. Untersuchungen der Befindlichkeit von Arbeitslosen in Deutschland, Schweden und den USA widerlegen nicht nur die Freiwilligkeit der Arbeitslosigkeit, sondern zeigen auch auf, dass die „freie“ Zeit für die Arbeitslosen ein „fatales Geschenk ${ }^{\text {“9 }}$ ist. Zum einen, weil die mit der Arbeitslosigkeit verbundenen materiellen Sorgen die Betroffenen stark belasten: Können die eingegangenen finanziellen Verpflichtungen eingehalten werden, kann die Familie, können die Kinder ausreichend versorgt werden? Ist die Altersversorgung gesichert? Über diese finanziellen Sorgen hinaus treten gravierende psychosoziale Probleme auf: Der Verlust sozialer Kontakte wird negativ empfunden, das Selbstwertgefühl beschädigt, die vermeintliche Nutzlosigkeit bedrückt, Ohnmachtsgefühle treten auf, gesundheitliche Schäden sind die Folge und die Suizidanfälligkeit steigt. Das Gewicht der drastischen Einkommenskürzung, die mit der vermeintlich üppigen Arbeitslosengeldzahlung verbunden ist, und die normalerweise auftretenden psychosozialen Probleme werden in der Bilanz des IAB wie von anderen Ökonomen häufig völlig vernachlässigt. Schon wegen des unterstellten generell arbeitsunwilligen Verhaltens der Arbeitslosen ist die Behauptung, dass der Arbeitsmarkt spürbar von Hartz IV profitiert habe, nicht nur kühn; sie kann nur falsch sein.

Sehr fraglich ist es auch, ob durch Druck und durch Verordnung existenzieller Not Eigenverantwortung und autonomes Handeln befördert werden können. Und ganz sicher muss bezweifelt werden, dass eine solche politische Maßnahme mit der Menschenwürde vereinbar ist, die auch jedem Arbeitslosen zusteht; zumal dann, wenn er für die Lage, in die er geraten ist, nicht verantwortlich ist. Massenarbeitslosigkeit geht nicht auf individuelles Verschulden, sondern auf eine Fehlfunktion des Wirtschaftssystems zurück. Ist es dann zu rechtfertigen, dass Arbeitslose für eine Fehlfunktion des Systems „bestraft" werden? Müssten nicht die Profiteure des fehlerhaften Systems die unverschuldet unter der Fehlfunktion Leidenden menschenwürdig alimentieren? ${ }^{10}$

\section{MANGEL AN OFFENEN STELLEN}

Hartz IV geht von einer zweiten unzutreffenden Annahme aus: Es wird unterstellt, dass genügend offene Stellen vorhanden seien, die von den unwilligen Arbeitslosen besetzt werden könnten, wenn ein entsprechender Druck auf sie ausgeübt würde. Die Relation der offenen Stellen zu der Zahl der Arbeitslosen macht aber deutlich, dass diese Annahme ganz und gar unrealistisch ist. Im Jahr der Verabschiedung des Hartz IVGesetzes betrug die Zahl der Arbeitslosen

4 Solow, R. (2007): Interview, in: Metallzeitung, November, S. 8f.; Niemeier, E. (2009): Ursachen der deutschen Wachstumsschwäche und Arbeitslosigkeit, in: Wirtschaftsdienst 9, S. 592ff

5 Klauder, W. (2005): Anhaltende Massenarbeitslosigkeit: richtige Diagnose und Politik?, in: Wirtschaftsdienst 7, S. 426

6 Möller et al. (2009): a.a.O., S. 1. Eine Gesamtbewertung der Arbeitsmarktwirkungen müsste auch die negativen Effekte auf das Lohnniveau insbesondere im Niedriglohnsektor und auf die Einkommensverteilung insgesamt umfassen. Sie haben zusätzliche Armut zur Folge und werden eine steigende Altersarmut in der Zukunft zur Folge haben. Sie sollen hier vernachlässigt werden, weil sie in der IAB-Untersuchung keine Rolle spielen.

7 Möller et al. (2009): a.a.O., S. 2, Tabelle 1.

8 Möller et al. (2009): a.a.O., S. 2

9 Kieselbach, T. (1990): Freie Zeit ist für Arbeitslose ein fatales Geschenk, in: Tribüne vom 29. 6. 1990

10 Die Beiträge zur Arbeitslosenversicherung wären dann durch Steuern zu ersetzen. 
6,06 Mio. (registriert 4,381 Mio., verdeckt 1,625 Mio.), die Zahl der gemeldeten offenen Stellen aber nur 286.000. ${ }^{11}$ Das heißt, dass die Zahl der Arbeitslosen 21fach höher lag als die Zahl der gemeldeten offenen Stellen. In den Jahren 2005 bis 2008 hat sich die Relation zwar vermindert, aber die Zahl der Arbeitslosen lag immer noch um ein Vielfaches über der Zahl offener Stellen. Selbst in der Aufschwungphase war es also nicht möglich, allen Arbeitslosen eine Stelle anzubieten. Noch viel weniger konnte dies in der Zeit geschehen, in der die HartzGesetze konzipiert wurden. Wenn aber die Zahl der Arbeitsplätze nicht ausreichte, konnte die Arbeitslosigkeit auch durch Druck auf Arbeitslose nicht vermindert werden. Mit der unzureichenden Verfügbarkeit ,adäquater“ Arbeitsplätze begründet das Institut übrigens, dass in der frühen Phase des SGB II (Ende 2005, Anfang 2006) die Beschäftigung durch Hartz IV nicht gestiegen sei. ${ }^{12}$ Damit bestätigt das IAB eigentlich, dass nicht die Aktivierung durch Hartz IV für den Anstieg der Beschäftigung verantwortlich ist, sondern das zusätzliche Angebot an Arbeitsplätzen, das durch den wirtschaftlichen Aufschwung ab Ende 2005 bewirkt wurde (vgl. den Anstieg der offenen Stellen ab 2005). Denn diese ,adäquaten"Arbeitsplätze, die zuvor nicht vorhanden waren, sind durch den wirtschaftlichen Aufschwung bereitgestellt worden.

Dass dieser wirtschaftliche Aufschwung für die positive Arbeitsmarktentwicklung eine Rolle gespielt hat, sprechen die IABForscher in ihrer Untersuchung durchaus an: „Die günstige Gesamtentwicklung fiel zwar in eine Phase des wirtschaftlichen Aufschwungs“". ${ }^{13}$ Dann jedoch relativieren sie diese Aussage: „Bestimmte Anzeichen sprechen aber dafür, dass der Rückgang der Arbeitslosenzahlen im SGB II nicht nur der positiven Konjunktur der Jahre 2006 bis 2008 geschuldet ist ${ }^{\text {" }}{ }^{14}$ Eine Differenzierung der Wirkungen nach konjunktureller und nach vermeintlicher Hartz-IV-Verursachung wird jedoch nicht vorgenommen. Die „bestimmten Anzeichen“, die dafür sprechen sollen, dass Hartz-IV positiv gewirkt habe, werden nicht genannt. Die IAB-Forscher meinen aber offenbar, einen „indirekten Hinweis“ in dem „empirischen Zusammenhang zwischen offenen Stellen und Arbeitslosigkeit, der sogenannten Beveridge-Kurve" zu sehen, der darauf hindeute, „dass infolge der Einführung des SGB II strukturelle Arbeitslosigkeit abgebaut werden konnte". ${ }^{15}$
$\mathrm{Zu}$ fragen ist, ob - was theoretisch möglich ist - wirklich strukturelle Arbeitslosigkeit in dem Maße vorherrschte und vorherrscht, die durch Hartz IV hätte abgebaut werden können. Dagegen spricht bereits die Vernachlässigung der Wirkungen der makroökonomischen Politik in der Problemanalyse rein angebotsorientierter Ökonomen. Kann trotzdem eine strukturelle oder institutionelle Arbeitslosigkeit festgestellt werden?

\section{STRUKTURELLE ARBEITSLOSIGKEIT?}

Strukturelle Arbeitslosigkeit liegt vor, wenn Angebot und Nachfrage nicht zusammenpassen, weil die beiden Aggregate hinsichtlich Qualifikation, Alter, Geschlecht, Region usw. unterschiedlich zusammengesetzt sind. Arbeitslosigkeit, die durch Arbeitsunwilligkeit entsteht, die - wie Ökonomen häufig formulieren - freiwillig ist, kann eigentlich nicht als echte strukturelle Arbeitslosigkeit bezeichnet werden. Die Zahl solcher unwilligen Arbeitslosen ist im Übrigen verhältnismäßig gering.

Zur strukturellen Arbeitslosigkeit ist generell festzustellen, dass der Übergang von ihr zur konjunkturellen Arbeitslosigkeit fließend und empirisch kaum abgrenzbar ist. Paul Krugman kritisierte schon 1999 die Versuchung, „einen wirtschaftlichen Abschwung als Zeichen ,struktureller Schwächen zu sehen, welche man erst beheben müsse, bevor eine Erholung möglich sei- oder zynischerweise gar zu argumentieren, es sei falsch, die Nachfrage zu beleben und eine Erholung einzuleiten, weil dies den Reformdruck verringern würde “" ${ }^{16}$ Auf diese Problematik einer nur vermeintlich strukturellen Arbeitslosigkeit weist auch der frühere IAB-Forscher Wolfgang Klauderhin, wenn er ausführt: „Nach allen Erfahrungen pflegt sich bei anhaltend hoher Arbeitsnachfrage selbst eine zuvor als strukturell klassifizierte Arbeitslosigkeit oft überraschend schnell zurückzubilden: durch weniger Entlassungen und weniger Neuzugänge in die Problemgruppen, durch mehr Umschulung, Weiterbildung und örtliche Mobilität oder durch Kettenmobilität, indem Beschäftigte aufsteigen und so Platz machen für geringer qualifizierte Arbeitslose “. ${ }^{17}$ Die Schwierigkeit, strukturelle Arbeitslosigkeit eindeutig erkennen zu können, ist also ein erster Grund, weshalb auch nicht-strukturelle politische Maßnahmen erfolgreich sein können und häufig erfolgreich sind. Ein zweiter Grund ist der, dass selbst strukturelle Probleme in der von Klauder beschriebenen Weise durch nachfragepolitische Maßnahmen, die die Arbeitskräftenachfrage steigern, bekämpft werden können.

\section{BEVERIDGE-KURVE BELEGT KEINE VERMINDERUNG STRUKTURELLER ARBEITSLOSIGKEIT}

Trotz der Schwierigkeit der Unterscheidung zwischen konjunktureller und struktureller Arbeitslosigkeit wird versucht, empirisch festzustellen, ob die strukturelle Arbeitslosigkeit steigt oder fällt. Ein Mittel dazu ist die Beveridge-Kurve, die offene Stellen und Arbeitslosigkeit zueinander in Beziehung setzt. Wenn Arbeitslosenzahl und offene Stellen die gleiche Höhe aufweisen, könnte die trotz offener Stellen bestehende Arbeitslosigkeit als strukturell bedingt angesehen werden. ${ }^{18}$ Im Koordinatensystem der Beveridge-Kurve ist dieser Fall bei gleicher Skalierung beider Achsen in allen Punkten gegeben, die auf der 45-Grad-Linie liegen. Nur in diesen Fällen kann man von struktureller Arbeitslosigkeit ausgehen. Wenn die Werte oberhalb der 45-Grad-Linie liegen, also mehr offene Stellen als Arbeitslose vorhanden sind, befindet sich die Volkswirtschaft in einer Phase der Hochkonjunktur. Wenn hingegen die Werte für Arbeitslosigkeit und offene Stellen unterhalb der 45-Grad-Linie liegen, es also mehr Arbeitslose als offene Stellen gibt, befindet sich die Volkswirtschaft in einer Schwächephase, in der es an Nachfrage nach Arbeitskräften und wahrscheinlich auch an gesamtwirtschaftlicher Güternachfrage mangelt. In den von der 45-Grad-Linie abweichenden Fällen ist es unmöglich festzustellen, wie hoch der eventuelle Anteil struktureller Arbeitslosigkeit ist! Eine Veränderung der strukturellen Arbeitslosigkeit lässt sich nur an dem Wert der Beveridge-Kurve auf der 45-Grad-Linie erkennen, der sich bei einer Verschiebung der Kurven unterschiedlicher Konjunkturzyklen ergibt. Eine Abnahme strukturel-

\footnotetext{
11 SVR (2007): a.a.O., S. 313 (Tabelle 47)

12 Möller et al. (2009): a. a. O., S. 4 f

13 Möller et al. (2009): a. a. O., S. 2

14 Möller et al. (2009): a. a. O., S. 2

15 Möller et al. (2009): a. a. O., S. 2

16 Krugman, P. (1999): Die große Rezession, Frankfurt/New York, S. 207

17 Klauder, W. (2005): a. a. O., S. 426

18 Kromphardt, J. (1998): Arbeitslosigkeit und Inflation, Göttingen, S. 51
} 
ler Arbeitslosigkeit ist dann zu vermuten, wenn sich die Kurve eines Konjunkturzyklus zum Ursprung des Koordinatensystems hin, also nach links verschiebt.

Die in der IAB-Untersuchung dargestellte Beveridge-Kurve verleitet die IAB-Forscher zu der Aussage, dass diese Kurve indirekt auf eine Verminderung der strukturellen Arbeitslosigkeit durch Hartz IV hindeute. Gegen diese Behauptung sind folgende Einwendungen zu erheben: Die im Kurzbericht dargestellte Beveridge-Kurve ist erstens in ein Koordinatensystem eingetragen, das unterschiedliche Maße für die Achsen aufweist: Die Arbeitslosen werden in Millionen, die offenen Stellen nur in Hunderttausenden gemessen. Dadurch wird optisch sowohl verdeckt, dass alle Werte - insbesondere auch die für 2008 und 2009 - unterhalb der 45-Grad-Linie liegen, d. h. dass sie auf eine Nachfrageschwäche hindeuten. Es wird ferner verdeckt, dass die Werte nicht auf der 45-Grad-Linie liegen, sodass eine Aussage über die strukturelle Arbeitslosigkeit gar nicht möglich ist. Eine Verbesserung der Relation offener Stellen zur Zahl der Arbeitslosen sagt eben nichts darüber aus, wodurch diese Verbesserung bewirkt wurde: ob durch einen Abbau eventueller struktureller Arbeitslosigkeit oder durch eine gestiegene Nachfragedynamik. Zweitens weist die dargestellte Kurve, wenn man sie denn kritiklos hinnähme, für die Jahre 2005 bis 2007 eine Rechtsverschiebung gegenüber der Kurve für den Konjunkturzyklus 1998 bis 2000 auf. Das heißt, dass diese Verschiebung nicht auf eine Verminderung, sondern auf eine Steigerung der strukturellen Arbeitslosigkeit hindeuten könnte. Erst die Werte für 2008 und 2009 liegen als Einzelwerte links von der Kurve des vorhergehenden Zyklus. Die Verschiebung dieser Einzelwerte 2008 und 2009 erklärt sich allerdings durch das besonders vorsichtige Entlassungsverhalten der Unternehmen, die in der gegenwärtigen Weltwirtschaftskrise das Instrument der Kurzarbeit ausgiebig nutzen und die wirkliche Arbeitslosigkeit dadurch unsichtbar machen.

Die dargestellte Beveridge-Kurve gibt also keinen Hinweis auf eine Verminderung der strukturellen Arbeitslosigkeit. Die einzig plausible Erklärungsmöglichkeit für den Abbau der Arbeitslosigkeit in den Jahren 2006 bis 2008 bietet die weltwirtschaftliche Nachfragedynamik, ergänzt durch demografische und Produktivitätsentwicklungen.

\section{FAZIT}

Das Ergebnis unserer Überlegungen lautet: Der Arbeitsmarkt hat - entgegen der Behauptung des IAB - durch Hartz IV nicht profitiert. Er konnte gar nicht profitieren, weil das Hartz-Konzept von einer falschen Verhaltensannahme ausging und bei der Analyse der Arbeitslosigkeit die Bedeutung einer falschen makroökonomischen Politik vernachlässigte. Ferner wäre Voraussetzung für die Wirksamkeit von Hartz IV gewesen, dass ausreichend offene Stellen vorhanden waren, die es in Wirklichkeit gar nicht gab. Arbeitsplätze werden nicht durch Druck auf die Arbeitslosen geschaffen, sondern durch die Ausweitung der Produktion, die ihrerseits durch eine Nachfrageausweitung bewirkt wird. Der Versuch, mithilfe der Beveridge-Kurve eine Verminderung der strukturellen Arbeitslosigkeit durch Hartz IV aufzuzeigen, misslingt, weil die Kurvenwerte in einem Koordinatensystem mit gleicher Skalierung unterhalb der 45-Grad-Linie liegen und deshalb gar keine Aussage über eine strukturelle Arbeitslosigkeit erlauben. Wenn man die dargestellte Kurve kritiklos hinnähme, würde die Kurvenverschiebung eher auf einen Anstieg der strukturellen Arbeitslosigkeit hinweisen. Die Steigerungen des BIP als Folge der dynamischen weltwirtschaftlichen Konjunktur in Verbindung mit der Produktivitätsentwicklung und der demografischen Entwicklung erklären dagegen schlüssig den Abbau der Arbeitslosigkeit. 